\title{
Sedação e Analgesia Pediátrica: comentários acerca das drogas utilizadas em procedimentos no pronto-socorro e os cuidados necessários antes, durante e após a sedoanalgesia
}

Amanda de Queiroz Germano Machadol ; Ana Caroliny de Castro Venâncio ${ }^{2}$; Francisca Priscila Sampaio Cruz Teles ${ }^{3}$; Mondeyv de Freitas Pascoal ${ }^{4}$; Jacquelinny Lopes de Macêdo ${ }^{5}$; Hermes Melo Teixeira Batista ${ }^{6}$

\begin{abstract}
Resumo: O principal objetivo da sedação em pediatria, está relacionado a necessidade de controlar o comportamento da criança ou adolescente, para que seja possível realizar um procedimento da forma mais segura possível. Especialmente em relação ao público infantil, cuja compreensão dos fatos é menor, a ansiedade e o medo podem causar dificuldade de se conseguir a colaboração do paciente. É importante que o local disponibilize de profissionais qualificados em sedoanalgesia pediátrica, como forma de evitar a dor imediata e possíveis traumas futuros no paciente.
\end{abstract}

Palavras-chave: Sedação, Analgesia, sedoanalgesia pediátrica.

\section{Pediatric Sedation and Analgesia: comments about the drugs used in procedures in the emergency room and the necessary care before, during and after the sedoanalgesia}

\begin{abstract}
The main objective of sedation in pediatrics is related to the need to control the behavior of the child or adolescent, so that it is possible to perform a procedure as safely as possible. Especially in relation to the child public, whose understanding of the facts is less, anxiety and fear can cause difficulty in getting the patient to collaborate. It is important that the place available of qualified professionals in pediatric sedoanalgesia, as a way to avoid immediate pain and possible future trauma in the patient.
\end{abstract}

Keywords: Sedation, Analgesia, pediatric sedoanalgesia.

\footnotetext{
${ }^{1}$ Graduada em Medicina pela Universidade de Fortaleza. Contato: amandaqgermano@gmail.com;

${ }^{2}$ Graduação em Medicina pela Faculdade Pernambucana de Saúde, Brasil;

${ }^{3}$ graduação em Medicina pela Faculdade De Medicina Estácio De Juazeiro Do Norte. Especialização - Residência médica pela Escola de Saúde Pública do Ceará, Brasil. Plantonista da Cirurgia Geral do Hospital e Maternidade São Francisco de Assis, Brasil;

${ }^{4}$ Cirurgião geral. Residência médica em Cirurgia Geral pela Escola de Saúde Pública do Estado do Ceará. Graduação em medicina pela Faculdade de Medicina Estácio de Juazeiro do Norte-CE;

${ }^{5}$ Graduação em Medicina pela Universidade Federal do Cariri, Brasil. Residente de Cirurgia Geral no Hospital Regional do Cariri;

${ }^{6}$ Graduação em Medicina pela Universidade Federal do Ceará. Médico do Hospital Regional do Cariri e Professor da Faculdade de Medicina de Juazeiro do Norte- Estácio. Doutorando em Ciências da Saúde pela FMABC, Médico do Hospital Universitário Júlio Bandeira - HUJB, Cajazeiras, PB. Contato: hermesmelo@oi.com.br.
} 


\section{Introdução}

Tanto para crianças quanto para adultos, o pronto socorro consiste em ambiente desagradável e potencialmente traumático. Especialmente em relação ao público infantil, cuja compreensão dos fatos é menor, a ansiedade e o medo podem se alastrar por ela, principalmente pelo temos da submissão à algum procedimento doloroso, motivo pelo qual é preciso agir no sentido de conseguir a colaboração do paciente.

Para procedimentos curtos e fáceis, é possível utilizar de técnicas de distração e relaxamento são suficientes, criando um ambiente propício de sedação consciente. Caso seja um procedimento mais demorado que necessite de imobilização, o uso de sedação farmacológica é indicado. No entanto, caso a tenha algum problema neurológico que diminuíam sua capacidade cognitiva para fins de colaborar com a sedação ou quando se tratar de procedimento doloroso, o uso da analgesia torna-se obrigatório.

Como é sabido, enquanto a analgesia é responsável por reduzir ou eliminar a percepção da dor pelo indivíduo, a sedação se preocupa em reduzir o estado de consciência do ser humano, onde muitos analgésicos também desencadeiam algum efeito sedativo, porém o inverso é bem raro. Ambos são utilizados para fins de diminuir a dor de um enfermo que precise se submeter a algum procedimento cirúrgico, e não raro a população infanto-juvenil necessita de sedação e analgesia para serem submetidos a algum procedimento de emergência, que atualmente podem ser realizados de variadas formas, sendo necessário a devida atenção quanto ao manejo adequado da substância por profissionais qualificados. Antes, esses procedimentos podiam ser invasivos ou não, mas em regra faziam parte de técnicas terapêuticas pediátricas que frequentemente eram desconfortáveis para a criança, os pais ou até mesmo para o profissional da saúde (KRAUSS; GREEN, 2006, MCCOY ET AL, 2016).

Hodiernamente, a sedação - antes procedimento exclusivo da anestesiologia - foi incorporada nas mais diversas especialidades da medicina, tais como gastroenterologia, neurologia, radiologia e medicina pediátrica (MASON, 2014, p. 48-62), no entanto, a padronização das práticas e uniformização das diretrizes e recomendações da sedação ainda se trata de um grande desafio (GOZAL; MASON, 2010).

O principal objetivo da sedação em pediatria está relacionado a necessidade de controlar o comportamento da criança ou adolescente para que seja possível realizar um procedimento da forma mais segura possível, o que difere do objetivo da sedação do paciente adulto, cuja finalidade principal é criar um estado de cooperação que permita 
tranquilidade e resposta aos comandos, pois é administrada para controlar o comportamento e permitir um término seguro do procedimento (MCCOY ET AL, 2016).

Nesse cenário, saber avaliar a dor da criança é de fundamental importância, sendo, inclusive, importante para verificar em que momento e a dose do analgésico deve ser ministrado. A severidade da sensação de dor deve ser avaliada individualmente e levando em conta as características de cada paciente, sendo essencial saber identificar quando é dor ou quando é ansiedade, para que não seja prescrita medicação inadequada.

Os principais componentes farmacológicos mais utilizados são os opióides, como cetamina e morfina; benzodiazepínicos, tais como midazolam, muito usado na pediatria), propofol, e cetamina e dexmedetomidina, para fins de se obter sedação e analgesia. Sendo essencial o conhecimento dos antagonistas dos opioides e dos benzodiazepínicos para que seja garantida a segurança da administração dessas drogas e seja possível diminuir as chances de adversidades.

Com base nisso, conforme será demonstrado o profissional responsável pelo manejo de protocolos de sedoanalgesia em procedimentos ocorridos em pronto-socorro de pediatria deve estar apto a realizar a avaliação do paciente e dos critérios necessário à devida escolha da medicação e do material adequado a garantir qualidade na assistência para fins de possibilitar o êxito da conclusão do procedimento.

\section{Sedação: breves comentários acerca da avaliação pré-sedativa e dos variados níveis de sedação}

A Sociedade Americana de Anestesiologia (ASA) recomenda que inicialmente deve ser realizada uma avaliação previamente ao início da sedação, com fulcro de identificar em que classificação se enquadra o paciente. A ASA (2002) dispõe de uma tabela classificatória classificação dos pacientes em seis categorias a depender de sua base salutar: 


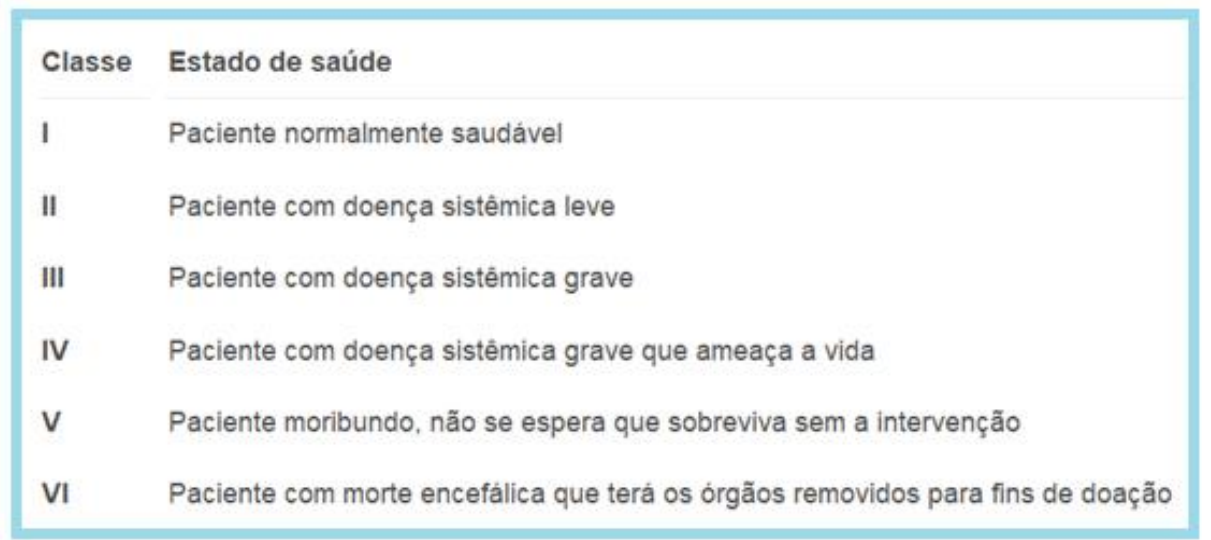

Conforme ensina a ASA (2002, p. 1004-1017), pacientes infanto-juvenis que se enquadrem na classificação I e II são apropriados a receber uma sedação mínima, moderada ou profunda; enquanto os que se enquadrem no padrão III e IV, que são pacientes com necessidades especiais ou alguma anormalidade anatômica nas vias aéreas, precisam de uma sedação de moderada a profunda.

Para fins de analisar em qual classificação se enquadra o paciente, os médicos podem do SAMPLE, acrônimo mnemônico que apresenta os componentes essenciais a serem avaliado no histórico médico na avaliação de pré-sedação:

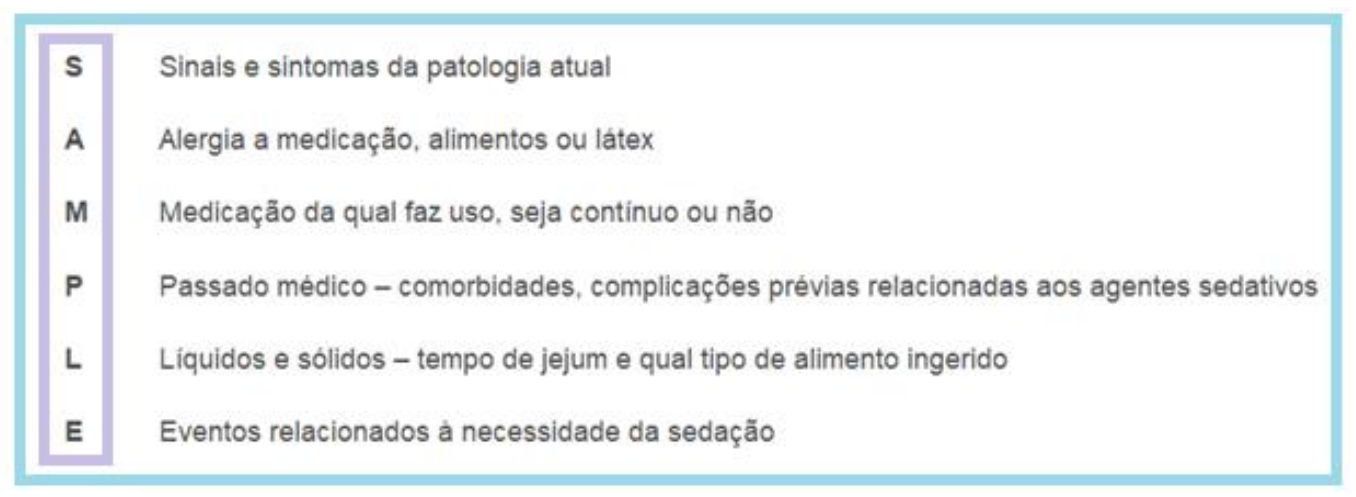

Com base nisso, cabe salientar que problemas adversos nas vias aéreas, sistema cardiovascular e respiratório, compõem as principais causas de mortalidade relacionadas à sedoanalgesia de doentes pediátricos (TOBIAS, 2011), isso ocorre porque os agentes sedativos possuem potencial de prejudicar os reflexos que atuam no sentido de proteger as vias aéreas. Assim, durante um procedimento de sedação, principalmente se profundo, deve-se estar atento para o respeito ao tempo necessário de jejum pré-operatório, bem como buscar agir com 
prudência, como forma de diminuir o risco da ocorrer uma aspiração pulmonar que prejudique o enfermo.

Nesse sentido, no momento do exame físico, deve-se atentar, em especial, para a existência de possível anormalidade cardíaca, pulmonar, renal, hepática ou genética, que dar ensejo a uma resposta inesperada da criança quando forem ministradas as medicações analgésicas e sedativas (BUTLER ET AL, 2000, JIRATIVANONT ET AL, 2017). Dessa forma, a análise das vias aéreas do paciente deve ser criteriosa, com vistas a identificar qualquer características capaz de aumentar o risco de obstrução durante o procedimento, tais como: micrognatia, hipertrofia significante de amígdalas, obesidade, pescoço curto, secreção em excesso ou diminuição dos reflexos de proteção das vias aéreas (ASA, 2012). As principais doenças genéticas e congênitas relacionadas com via aérea de difícil manejo são: (i) a fenda labial e palatine, (ii) trissoma do cromossomo 21, (iii) fibroplastia ossificante progressiva, (iv) mucopolissacardiose, (v) malformações vasculares, (vi) Síndrome de Pierre Robin, (vii) Síndrome de Crouzon, (viii) Síndrome de Treacher-Collins, (ix) Síndrome de Goldenhar, (x) Síndrome de Klippel-Feil e (xi) Síndrome de Freeman-Sheldon (BUTLER ET AL, 2000, p. $837-855)$.

Uma análise realizada com cerca de 11 mil crianças, todas submetidas a procedimentos com uso de anestesia geral e intubação traqueal, verificou que a laringoscopia foi o procedimento mais difícil dentre os casos, com percentuais mais altos em crianças com menos de um ano de idade alcançando $4,7 \%$ e $0,7 \%$ em crianças com mais de um ano. Ademais, identificou-se que que as crianças enquadradas na classificação III e IV definida pela ASA e com IMC baixo, possuem mais risco de passar por uma laringoscopia difícil, se submetidos a procedimentos cirúrgicos na cavidade oral, maxilofaciais ou cardíacaos (HEINRICH ET AL, 2012, p. 729-736).

Nesse diapasão, para fins de evitar quaisquer problemas maiores, os sinais vitais da criança enferma devem ser registrados antes de dar-se início a sedação, após a aplicação de cada dose de medicação, em intervalos de tempo no decorrer o procedimento até o término, na fase de recuperação, bem como por ocasião da alta (ASA, 2002). Ademais, a monitoração do doente deve ser ininterrupta com uso de oxímetro de pulso e monitor dos batimentos cardíacos, ritmo respiratório e de pressão arterial, exceto no caso de sedação mínima, na qual observação do nível de consciência da criança resta suficiente (COTÉ; WILSON, 2016).

Outrossim, deve-se dispender uma atenção especial caso seja necessário cobrir a face e/ou tronco da criança, visto que é de suma importância observar a coloração de suas mucosas 
e o movimento da caixa torácica. Inclusive, caso se trate de situação em que a observação esteja prejudicada, tal como ocorre na ressonância magnética, recomenda-se o uso de equipamento de monitoração não invasiva e contínua. Por fim, além das fontes usuais de oxigênio e material de sucção, é obrigatório que no carrinho de emergência sempre tenha um desfibrilador, as drogas necessárias em caso de ressuscitação, antídotos adequados e equipamentos utilizados para vias aéreas difíceis (ASA, 2002).

Nesse sentido, é incontroversa a importância da presença de profissionais qualificados e devidamente treinados para fins de reconhecer possível comprometimento da via aérea da criança, e intervir de forma adequada e a tempo. Assim, recomenda-se que estejam presentes profissionais especializados em pediatria para monitorar, manejar e succionar as vias aéreas se necessário, com uso de ventilação manual, acesso vascular e ressuscitação cardiopulmonar, etc (AAP, 2016), isso porque a presença de profissionais qualificados reduz os inconvenientes das adversidades, bem como aumenta a sensação de conforto e segurança do enfermo (SAUTER ET AL, 2016, p. 97, ZAVERI ET AL, 2016, p. 486).

A sedação pode ser definida como um ato contínuo composto por estágios progressivos que podem ir de uma sedação leve até à anestesia geral. Com base nisso, em 2002, a ASA definiu quatro principais níveis de sedação, sendo eles: (i) mínima (ansiólise), na qual a medicação ministrada não impossibilita que o enfermo responda normalmente aos comandos verbais, embora a cognição e coordenação estejam prejudicadas; (ii) moderada ou consciente, em que cria-se um estado de consciência induzido por medicamentos, em que o paciente é capaz de responder propositalmente à comandos verbais, de forma isolada ou acompanhada de estímulos táteis; (iii) profunda, onde as drogas utilizadas impedem que o paciente seja facilmente acordado, mas são capazes de responder à estímulos repetidos ou dolorosos, e pode ser necessário auxílio para manter a cadência da função ventilatória; e (iv) anestesia geral, que é um estado de perda de consciência total induzida por meio do uso de medicamentos para que o enfermo não seja capaz de ser despertado, nem com estímulos repetidos, tampouco dolorosos. $\mathrm{Na}$ anestesia geral tanto a respiração quanto a função cardiovascular podem ser prejudicadas, carecendo de auxílio para se manterem.

Sem dúvidas, evitar a dor é um dos principais objetivos da sedoanalgesia pediátrica, sendo necessário, para se obter êxito na mitigação dos estímulos dolorosos, que a criança seja devidamente analisada. A dor em uma criança pode ser verificada por meio de características fisiológicas, observação de seus comportamentos, bem como do próprio relato do paciente. 
Em regra, um enfermo com dor apresenta sintomas específicos relacionados com taquicardia, dilatação das pupilas, sudorese e vasoconstricção periférica (BÜTTNER; FINKE, 2000, p. 303-318, GONSALVES; MERCER, 1993, p. 88-93). Contudo, a avaliação não se resume a esses parâmetros, sendo importante analisar outros sinais a depender da fase de desenvolvimento da criança e a sua capacidade de relatar a dor (CRELLIN ET AL, 2007).

A título de exemplo, a Neonatal Infant Pain Scale (NIPS) apresenta possíveis escalas e resposta à estímulos dolorosos em crianças em estágio neonatal, com base em alguns parâmetros objetivos, onde a dor é verificada se o somatório for maior que três pontos:

\begin{tabular}{|c|c|c|c|}
\hline Indicador & 0 ponto & 1 ponto & 2 pontos \\
\hline Expressão facial & Relaxada & Contraida & - \\
\hline Choro & Ausente & Resmungos & Vigoroso \\
\hline Respiração & Regular & Diferente da basal & - \\
\hline Braços & Relaxados & Fletidos/estendidos & -. \\
\hline Pernas & Relaxadas & Fletidas/estendidas & -- \\
\hline Estado de alerta & Dormindo e/ou calmo & Agitado e/ou irritado & -. \\
\hline
\end{tabular}

Fonte: LAWRENCE ET AL, 1993, p. 59-66.

Da mesma forma a escala FLACC, em português face, pernas, atividade, choro e consoabilidade , é utilizada para avaliar crianças de dois meses a sete anos de idade, por meio da análise de cinco reações a estímulos dolorosos, em uma escala de 0 a 2 :

\begin{tabular}{|c|c|c|c|}
\hline Categorias & 0 ponto & 1 ponto & 2 pontos \\
\hline Face & $\begin{array}{l}\text { Nenhuma } \\
\text { expressão } \\
\text { particular ou } \\
\text { sorriso }\end{array}$ & $\begin{array}{l}\text { Caretas ou franzir de } \\
\text { sobrancelhas ocasionais, } \\
\text { introversão, desinteresse }\end{array}$ & $\begin{array}{l}\text { Caretas ou franzir de } \\
\text { sobrancelhas frequentes, } \\
\text { queixo tremendo, mandibula } \\
\text { cerrada }\end{array}$ \\
\hline Pernas & $\begin{array}{l}\text { Posição normal } \\
\text { ou relaxada }\end{array}$ & Inquietas, agitadas, tensas & Chutando ou esticadas \\
\hline Atividade & $\begin{array}{l}\text { Quieta, posição } \\
\text { normal, move-se } \\
\text { facilmente }\end{array}$ & $\begin{array}{l}\text { Contorcendo-se, movendo-se } \\
\text { para frente e para trás, tensa }\end{array}$ & $\begin{array}{l}\text { Curvada, rigida ou com } \\
\text { movimentos bruscos }\end{array}$ \\
\hline Choro & $\begin{array}{l}\text { Sem choro } \\
\text { (acordada ou } \\
\text { dormindo) }\end{array}$ & $\begin{array}{l}\text { Gemidos ou choramingos, } \\
\text { queixa-se ocasionalmente }\end{array}$ & $\begin{array}{l}\text { Choro continuo, grito ou } \\
\text { soluço; queixa-se com } \\
\text { frequência }\end{array}$ \\
\hline Consolabilidade & $\begin{array}{l}\text { Satisfeita, } \\
\text { relaxada }\end{array}$ & $\begin{array}{l}\text { Tranquilizadas por toques, } \\
\text { abraços ou conversas: } \\
\text { possivel de distrair }\end{array}$ & Dificil de consolar ou confortar \\
\hline
\end{tabular}

Fonte: MERKEL ET AL, 1997, p. 293-297. 
Em crianças menores, é comum a análise da face, tendo em vista que essas não possuem a habilidade de explicar a intensidade de dor de maneira adequada. Assim, uma escala facial facilita o reconhecimento da ausência ou do nível da dor que o enfermo está sentindo. Nesse método o profissional deve apresentar uma imagem em que esteja disposta uma escala facial de dor e tentar pedir que a criança aponte em qual ela se enquadra:

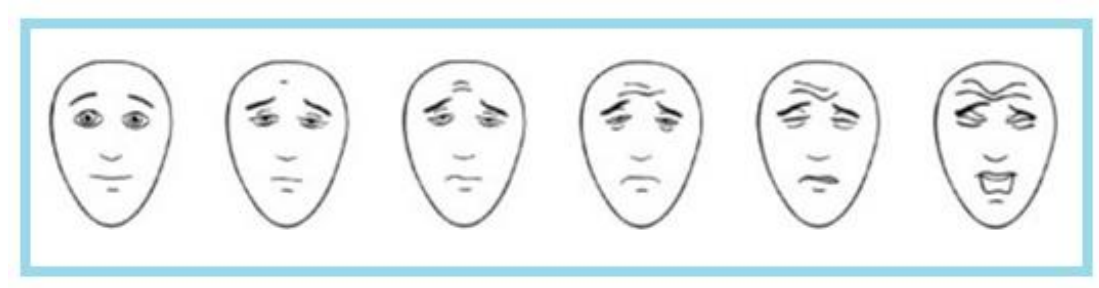

Fonte: BIERI; REEVE, 1990.

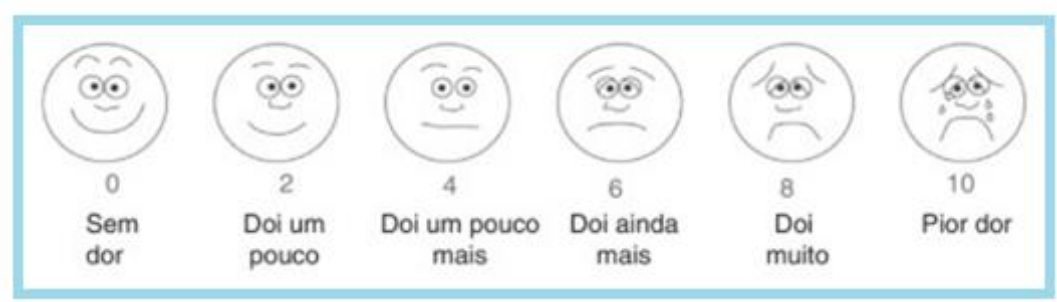

Fonte: GARRA ET AL, 2010, p. 50-54.

Nesse contexto, cabe comentar acerca de quadro elaborado por McGrath (1995) em relação aos fatores que interferem na percepção de dor da criança, uma vez que essa análise deve ser cuidadosa, já que, assim como ocorre com adultos, a presença de dor sem lesão aparente pode ocorrer pelos mais variados motivos: 


\begin{tabular}{|c|c|c|}
\hline $\begin{array}{l}\text { COGNITIVA } \\
\text { Compressão } \\
\text { Controle } \\
\text { Expectativa } \\
\text { Relevância } \\
\text { Estratégias de controle da dor }\end{array}$ & $\begin{array}{l}\text { COMPORTAMENTAL } \\
\text { Ações em público } \\
\text { Respostas dos pais/equipe } \\
\text { Restrição fisica } \\
\text { Atividades fisicas } \\
\text { Atividades sociais }\end{array}$ & $\begin{array}{l}\text { EMOCIONAL } \\
\text { Ansiedade } \\
\text { Medo } \\
\text { Frustração } \\
\text { Raiva } \\
\text { Depressão }\end{array}$ \\
\hline Lesão tecidual & $\begin{array}{l}\text { Idade } \\
\text { Raça } \\
\text { Nivel cognitivo } \\
\text { Experiências prévias de dor } \\
\text { Aprendizado familiar } \\
\text { Cultural }\end{array}$ & Sensação de dor \\
\hline
\end{tabular}

Todo e qualquer cuidado é importante quando se trata de sedação e a analgesia, principalmente em se tratando de pacientes pediátricos, tendo em vista que quanto mais jovem a criança ou mais enferma, mais profunda deve ser a sedação, razão pela qual a análise prévia da situação do enfermo resta imperiosa, para que o profissional seja capaz de escolher a adequadamente a droga, as doses e a melhor forma de administrá-la.

\section{Principais drogas a serem ministradas em pacientes pediátricos}

A escolha da medicação deve considerar critérios correlacionados ao que o enfermo será submetido e ao quadro de base e comorbidades correlato. A seguir listaremos um breve resume das principais drogas e suas principais características e utilidades:

\begin{tabular}{|c|c|c|c|}
\hline Droga & Efeitos & Efeito colateral & Indicações \\
\hline & \multicolumn{2}{|c|}{ Sedativo-hipnóticas } & \\
\hline Midazolam & $\begin{array}{l}\text { Sedação, controle } \\
\text { motor, redução de } \\
\text { ansiedade, sem } \\
\text { efeito analgésico }\end{array}$ & $\begin{array}{l}\text { Hipotensão, } \\
\text { depressão } \\
\text { respiratória, } \\
\text { efeito paradoxal }\end{array}$ & $\begin{array}{l}\text { Procedimento } \\
\text { que requerem } \\
\text { sedação, } \\
\text { Redução da } \\
\text { ansiedade ou } \\
\text { amnésia }\end{array}$ \\
\hline
\end{tabular}




\begin{tabular}{|c|c|c|c|}
\hline Diazepam & $\begin{array}{l}\text { Sedação, controle } \\
\text { motor, redução de } \\
\text { ansiedade, sem } \\
\text { efeito analgésico }\end{array}$ & $\begin{array}{l}\text { Hipotensão, } \\
\text { depressão } \\
\text { respiratória, } \\
\text { efeito paradoxal }\end{array}$ & $\begin{array}{l}\text { Pouco usado } \\
\text { devido à meia } \\
\text { vida longa }\end{array}$ \\
\hline Propofol & $\begin{array}{l}\text { Sedação rápida e } \\
\text { curta }\end{array}$ & $\begin{array}{l}\text { Pode causar dor } \\
\text { à infusão, } \\
\text { hipotensão, } \\
\text { apneia e } \\
\text { bradicardia }\end{array}$ & $\begin{array}{l}\text { Procedimento } \\
\text { de curta } \\
\text { duração, } \\
\text { associados o। } \\
\text { não a } \\
\text { analgésicos }\end{array}$ \\
\hline Dexmedetomidina & $\begin{array}{l}\text { Sedação com } \\
\text { manutenção do } \\
\text { drive respiratório } \\
\text { Não altera traçado } \\
\text { do EEG } \\
\text { Uso off label na } \\
\text { pediatria }\end{array}$ & $\begin{array}{l}\text { Pode causar } \\
\text { arritmia, } \\
\text { hipotensão e } \\
\text { hipertensão }\end{array}$ & $\begin{array}{l}\text { Procedimento } \\
\text { de imagem } \\
\text { (TC e RNM), } \\
\text { endoscopia, } \\
\text { EEG }\end{array}$ \\
\hline
\end{tabular}

Analgésicos

\begin{tabular}{|c|c|c|c|}
\hline Fentanil & Analgesia & $\begin{array}{l}\text { Bradicardia, } \\
\text { rigidez torácica, } \\
\text { depressão } \\
\text { respiratória }\end{array}$ & $\begin{array}{l}\text { Procedimento } \\
\text { com dor } \\
\text { moderada a } \\
\text { severa }\end{array}$ \\
\hline Morfina & Analgesia & $\begin{array}{l}\text { Liberação de } \\
\text { histamina, } \\
\text { hipotensão, } \\
\text { náuseas, } \\
\text { redução da } \\
\text { motilidade } \\
\text { gastrointestinal }\end{array}$ & $\begin{array}{l}\text { Procedimento } \\
\text { com dor } \\
\text { moderada a } \\
\text { severa }\end{array}$ \\
\hline Cetamina & $\begin{array}{l}\text { Agende } \\
\text { dissociativo com } \\
\text { propriedades } \\
\text { analgésicas e } \\
\text { sedativas }\end{array}$ & $\begin{array}{l}\text { Laringoespasmo, } \\
\text { hipersalivação, } \\
\text { reações de } \\
\text { emergência, } \\
\text { vômitos }\end{array}$ & $\begin{array}{l}\text { Procedimento } \\
\text { dolorosos de } \\
\text { curta duração } \\
\text { ou quando a } \\
\text { amnésia é } \\
\text { desejada }\end{array}$ \\
\hline
\end{tabular}




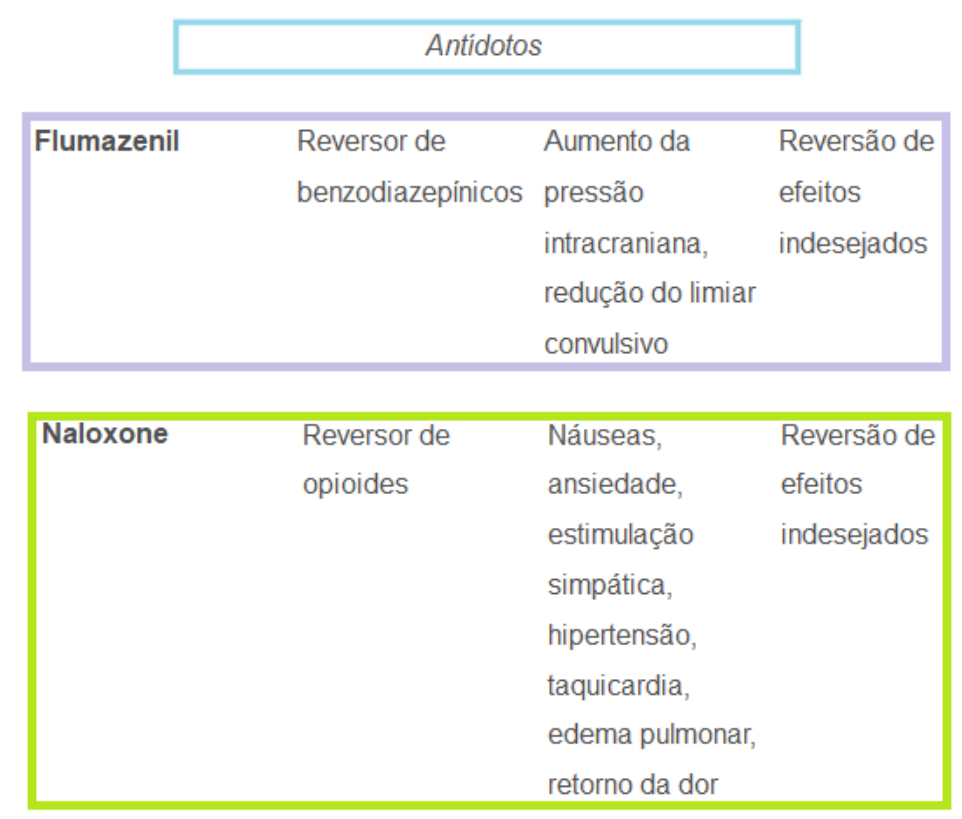

Como pode se verificar, as principais drogas utilizadas: são benzodiazepínicos (diazepan, midazolan), opioides (morfina, fentanil,), cetamina, propofol e dexmedetomidina.

Os benzodiazepínicos substâncias sedativas hipnóticas, cujo mecanismo de ação atua inibindo o sistema nervoso central (SNC) e seus efeitos farmacológicos são a sedação, hipnose, diminuição de ansiedade, amnésia, relaxamento da musculatura e anticonvulsivo. Não desencadeia efeito analgésico, razão pela qual deve ser associado a outras substâncias em caso de procedimentos dolorosos. Dentre os principais benzodiazepínicos utilizados em procedimentos de sedoanalgesia pediátrica, destacamos o diazepam e o midazolam.

O primeiro é muito utilizado para fins sedativos, no entanto sua ministração pode da ensejo à efeitos muito prolongados, tendo em vista que possui uma meia vida longa e variável. Em razão dessa variabilidade, seu uso tem sido substituído pelo midazolam, pois a variabilidade de sua administração é de menor duração (KRAUSS, 2006). Inclusive, estudo comparativo entre diazepam e midazolan, analisando a utilização das drogas em procedimentos de emergência, revelou que enfermos que receberam o midazolam obtiveram um nível mais elevado de sedação precoce, necessidade reduzida de renovação da dose durante o procedimento e menor sensação de dor durante a infusão (Wright et al, 1993, p. 201-205).

Devido a isto, conforme demonstrou pesquisa realizada no Brasil por Sukys et al (2011, p. 343-349), o midazolam tem sido atualmente a droga mais amplamente utilizada nas emergências pediátricas, sendo o sedativo de escolha em $80 \%$ dos eventos que careciam de intubação rápida, tendo em vista que possui rápido início de ação, curta duração, e boa 
variedade de vias de administração, podendo ser ministrado por via oral, retal, intranasal, intramuscular ou intravenosa.

Como medicamento utilizado para fins de reverter os efeitos dos benzodiazepínicos de ação central, utiliza-se o flumazenil, medicamente de administração exclusivamente intravenosa. É interessante comentar que para a reversão dos efeitos é preciso utilizar quantidade de flumazenil contrária ao seu aparecimento durante a sedação, assim, são necessárias altas doses de flumazenil para reverter o efeito do sedativo de pequenas doses de midazolam, e pequenas doses do flumazenil revertem o efeito de altas doses do midazolam.

Os opioides é um grupo de drogas que atuam diretamente nos receptores opioides neuronais com o objetivo de modular a percepção cortical da dor de moderada a grave.

Os principais opioides utilizados no pronto-socorro infanto-juvenil são a morfina e fentanil.

A morfina trata-se de substância que possui um início de ação demorado, em razão de de sua solubilidade lipídica relativamente baixa, e uma duração prolongada. O metabolismo de primeira passagem é bem significativo, devendo ser aplicadas doses seis vezes maiores do que as doses parenterais para que seja possível alcançar o mesmo grau de analgesia (STANSKI; GREENBLATT; LOWENSTEIN, 1978, p. 52-59). A morfina é indicada para a procedimentos em que é necessária uma analgesia prolongada, bem como em caso de necessidade de se aliviar dor intensa aguda ou crônica; e sua descontinuação necessita de fenômenos de abstinência, cujos sinais incluem dilatação da pupila, lacrimejamento, suor, arrepios, hipertensão, febre, vômito, dores musculares e articulares, alterações de comportamentos e diarreia (TOBIAS, 2000, p. 2122-2132).

Outro opioide muito utilizado na analgesia pediátrica é o fentanil, sendo este um opioide sintético cem vezes mais potente que a morfina e com ação muito mais rápida devido a sua alta lipossolubilidade. Ademais, estudos têm apontado que é possível administrar o fentanil por outras vias, sendo possível obter, da mesma forma, a sua eficiência para o alívio da dor.

Estudo comparativo entre fentanil e morfina aplicado em enfermos pediátricos para fins de redução de fraturas, demonstrou que fentanil intranasal na dose de $1,7 \mathrm{mcg} / \mathrm{Kg}$, possui efeitos semelhante a aplicação de morfina intravenosa na dose de $0,1 \mathrm{mg} / \mathrm{Kg}$ (BORLAND ET AL, 2001, p. 202-208). Corroborando com esse estudo, resultados semelhantes foram obtidos por por Young et al (1999, p 90-94), ao comparar o uso de fentanil intranasal, na dose de $1 \mathrm{mcg} / \mathrm{Kg}$, com morfina instramucular, na dose de $0,2 \mathrm{mg} / \mathrm{Kg}$, para o mesmo procedimento pediátrico. 
Deve-se ressaltar a depressão respiratória consiste no efeito colateral mais decorrente no uso dos opioides, estando diretamente relacionada com dose aplicada ao paciente. Essa depressão respiratória é relativamente maior quando os opioides são ministrados combinados com substâncias benzodiazepínicas. Isso foi comprovado por estudos realizados com 2.500 pacientes submetidos a sedação e analgesia em uma emergência pediátrica nos EUA, que revelou que 19,3\% dos eventos adversos respiratórios haviam sido causados pela associação de midazolam e fentanil, enquanto apenas 5,8\% decorreu do uso isolado de midazolam (Roback et al, 2005, p. 508-513).

Como antítodo dos opioides, o naloxone é a substância mais utilizada. Deve ser administrada de forma intravenosa e intramuscular, de preferência, podendo também ser aplicado por vias subcutânea, sublingual e endotraqueal. A dose depende do efeito desejado, e podem ser repetidas a cada dois minutos até que o efeito esperado seja obtido, já que a meiavida desse antídoto é menor do que da morfina e do fenatil. Pode causar náuseas, ansiedade, estimulação do sistema simpático, hipertensão, taquicardia, edema de pulmão e dor. No entanto, estudos apontam no sentido de que, se aplicado em pequenas doses, o naloxone é capaz de reduzir as náuseas causadas pelos opioides e os escores de dor. (BARRONS; WOODS, 2011).

A cetamina também é substância muito utilizada para a analgesia em pronto-socorro de crianças. Trata-se de agente anestésico que pode desencadear um estado dissociativo devido ao bloqueio entre as funções límbicas e as funções córtico-talâmicas. Causa amnésia e analgesia, sendo eficaz em procedimentos dolorosos, tais como como queimaduras e reduções de fraturas. Preserva o tônus e as vias aéreas superiores desobstruídas e estabilidade cardiovascular, apesar de causar aumento da frequência cardíaca e da pressão arterial a depender da dose utilizada, em razão de estímulo simpático.

Indica-se o uso da cetamina para procedimentos potencialmente dolorosos e de curta duração ou em casos que se deseja a amnésia da criança, como testes realizados para fins de verificar se houve abuso físico (Green; Roback; Kennedy, 2001). É administrada por meio de via intravenosa, intramuscular, intranasal e oral. Pode ter como efeitos adversos laringoespasmo, apneia transitória, vômito e agitação; ademais, como inibe a recaptação de catecolaminas, pode ocasionar o aumento da pressão arterial, da frequência cardíaca e/ou débito cardíaco.

A cetamina é contraindicada para pacientes com menos de três meses de idade devido as possíveis complicações das vias aéreas, bem como em enfermos esquizofrênicos, pois pode causar estímulo psicótico; deve ser evitado em portadores de cardiopatas, com patologias 
pulmonares e/ou anomalias do sistema nervoso central; e é contraindicado para pacientes com alteração anatômica intracraniana e hidrocefalia (LAHTI; KOFFEL; LAPORTE, 1995, p. 9-19; GREEN, ANDOLFATTO, KRAUSS, 2015, p. 52-54), pois pode causar aumento da pressão intracraniana.

No último grupo de principais drogas sedativas-hipnóticas utilizadas em emergências de pediatria, temos o propofol e o Dexmedetomidina. O primeiro possui ação rápida e de curta duração de efeito, indicado para procedimentos breves, podendo ser associado à drogas analgésicas, tais como fentanil e cetamina. (GODAMBE ET AL, 2003). É de fácil titulação, pouco acúmulo e baixos efeitos colaterais. Sobre essa droga, estudo realizado com 200 crianças enfermas que iriam se submeter a exames de imagem, por Sebe et al (2014, p. 225-230), demonstrou que o uso do propofol é mais eficaz do que a administração de midazolam para fins sedativos e devido ao menor tempo de recuperação. Ressalte-se que, em caso de procedimentos de imagem mais complexos e morosos, a droga deve ser utilizada de forma contínua para manter seus efeitos, sem que isso comprometa a recuperação ou possíveis efeitos adversos (GRIFFITHS et al, 2013). Apesar de possuir alguns efeitos adversos esperados, o uso da substância é seguro quando realizado com o devido monitoramento.

Por fim, a dexmedetomidina é capaz de promover a sedação sem diminuição do ritmo respiratório. No entanto, seu uso ainda é off-label na pediatria, ou seja, não é aprovado e não consta da bula, motivo pelo qual sua utilização é feita por conta e risco do médico. Apesar disso, seu uso tem crescido em procedimentos pediátricos de emergência, podendo ser associado ao midazolam, cetamina ou algum opioide (MASON; LERMAN, 2011). Pode ser administrado de forma intravenosa, intranasal, oral e gástrica; e seus efeitos adversos envolvem hipotensão, bradicardia e arritmia. (CARNEY; KENDRICK, 2013, p. 21-27).

\section{Considerações finais}

A sedação e a analgesia em pediatria demonstram sua importância por serem métodos essenciais para facilitar a realização de um procedimento de emergência, bem como para a execução de procedimentos potencialmente dolorosos. Nesse meio, a escolha correta da droga a ser utilizada demasiadamente relevante, principalmente porque carece de uma pré-avaliação minuciosa das necessidades individuais de cada paciente, tendo em vista as particularidades do corpo humano, não havendo um protocolo rígido a ser aplicado. 
O próprio ambiente de pronto socorro já configura situação de ansiedade e medo, motivo pelo qual deve-se buscar amenizar esses sentimentos nas especialmente em se tratando de crianças, não só com vistas a facilitar a execução de procedimentos, como também para evitar possíveis traumas. Assim, resta imperioso saber manejar essas sensações nas crianças, tanto fora quanto durante procedimentos em sala cirúrgica, permitindo, inclusive, que as crianças cooperativas sejam submetidas a métodos mais simples, como hipnose, distração e anestésicos tópicos, caso configure situação que afaste a necessidade de sedação farmacológica.

Nesse contexto, é importante ressaltar que não existe um consenso acerca do melhor sedativo ou analgésico a ser manejado em procedimentos dolorosos na emergência pediátrica, motivo pelo qual a análise da dor na medicina pediátrica deve observar o grau de desenvolvimento da criança e sua autonomia, isto porque a inabilidade das crianças de quantificar e qualificar os estímulos dolorosos que estão sentindo, são as principais causas da progressão de injúrias ou desenvolvimento de medos por eventos adversos.

Conforme comentado, os procedimentos de sedoanalgesia necessitam te uma equipe treinada e bem qualificada, que saiba escolher as drogas mais adequadas a cada caso, levando em consideração as particularidades fisiológicas e psicológicas do paciente a idade. Com base nisso, havendo a necessidade de se executar procedimentos dolorosos na urgência e emergência pediátrica, é importante o uso da analgesia e sedação, a ser escolhido de forma criteriosa após a avaliação do paciente, para que seja possível a qualidade na assistência da criança, razão pela qual a sedoanalgesia tem sido uma prática cada vez mais utilizada em pronto-socorro pediátrico.

Por fim, urge comentar que apesar da importância da diminuição ou redução da dor em procedimento de pronto socorro pediátrico, não existe a melhor forma de realiza-los, não apenas devido as particularidades fisiológicas de cada criança, mas principalmente em razão da dificuldade que possuem em expressar sua dor. Assim, é importante que o local disponibilize de profissionais qualificados em sedoanalgesia pediátrica, como forma de evitar a dor imediata e possíveis traumas futuros no paciente.

\section{Referências}

AMERICAN SOCIETY OF ANESTHESIOLOGISTS. Task Force on Sedation and Analgesia by Non-Anesthesiologists. Practice guidelines for sedation and analgesia by non-anesthesiologists. Anesthesiology, 96, 2002. 
BARTOLOME, S.M.; CID, J.L.; FREDDI, N. Analgesia and sedation in children: practical approach for the most frequent situations. J Pediatr (Rio J), 83, 2007.

BATALGA, L. M. C. B.; CARREIRA, M. C. G.; CORREIA, M. M. C. Dor para não ter dor: aplicação de anestésico tópico. Rev. Enf. Ref. Vol. 3(5), Coimbra, dez, 2011.

BEYER, J.E.; ARADINE, C.R. Content validity of an instrument to measure young children's perceptions of the intensity of their pain. J Pediatr Nurs, 1, 1986.

BORLAND, M.; MILSON, S.; ESSON, A. Equivalency of two concentrations of fentanyl administered by the intranasal route for acute analgesia in children in a paediatric emergency department: a randomized controlled trial. Emerg Med Aust, 23, 2011.

BUTLER, M.G.; HAYES, B.G.; HATHAWAY, M.M.; BEGLEITER, M.L. Specific genetic diseases at risk for sedation/anesthesia complications. Anesth Analg, 91, 2000.

BÜTTNER, W.; FINKE, W. Analysis of behavioural and physiological parameters for the assessment of postoperative analgesic demand in newborns, infants and young children: a comprehensive report on seven consecutive studies. Paediatr Anaesth, 10, 2000.

CARNEY, L.; KENDRICL, J.; CARR, R. Safety and effectiveness of dexmedetomidine in the pediatric intensive care unit (SAD-PICU). Can J Hosp Pharm, 66, 2013

CARVALHO, W. B.; TROSTER, E.J. Sedação e analgesia para procedimentos no pronto-socorro de pediatria. J Pediatr (Rio J), 75, 1999.

CHIARETTI, A.; BARONE, G.; RIGANTE, D.; RUGGIERO, A.; PIERRI, F.; BARBI, E. Intranasal lidocaine and midazolam for procedural sedation in children. Arch Dis Child, 96, 2011.

COTÉ, C. J.; WILSON, S. Guidelines for monitoring and management of pediatric patients before, during, and after sedation for diagnostic and therapeutic procedures: update 2016, Pediatrics, 138, 2016.

CRELLIN, D.; SULLIVAN, T.P.; BABL, F. E.; O'SULLIVAN, R.; HUTCHINSON, A. Analysis of the validation of existing behavioral pain and distress scales for use in the procedural setting. Paediatr Anaesth, 17, 2007.

GARRA, G.; SINGER, A. J.; TAIRA, B. R.; CHOHAN, J.; CARDOZ, H.; CHISENA, e. Validation of the Wong-Baker FACES Pain Rating Scale in pediatric emergency department patients. Acad Emerg Med, 17, 2010.

GODAMBE, S.A.; ELLIOT, V.; MARGENY, D.; PERSHAD, J. Comparison of propofol/fentanyl versus ketamine/midazolam for brief orthopedic procedural sedation in a pediatric emergency department, Pediatrics, 112, 2003.

GOZAL, D.; MASON, K.P. Pediatric sedation: a global challenge. Int J Pediatric, 2010.

GRIFFITH, N.; HOWELL, S.; MASSON, D.G. Intranasal midazolam for premedication of children undergoing day-case anesthesia: comparison of two delivery systems with assessment of intraobserver variability. Br J Anesth, 81, 1998. 
GRIFFITHS, M.A.; KAMAT, P.P.; MCCRACKEN, C.E.; SIMON, H.K. Is procedural sedation with propofol acceptable for complex imaging? A comparison of short vs. prolonged sedations in children. Pediatr Radiol, 43, 2013.

KRAUSS, B; GREEN, S.M. Procedural sedation and analgesia in children. Lancet, 367, 2006.

KRAUSS, B. S.; KRAUSS, B.A.; GREEN, S.M. Procedural sedation and analgesia in children. Engl J Med, 370, 2014.

LABROO, R.B; PAINE, M.F.; THUMMEL, K.E.; KHARASCH, E.D. Fentanyl metabolism by human hepatic and intestinal cytochrome P4503A4: implications for interindividual variability in disposition, efficacy, and drug interactions. Drug Metab Dispos, 25, 1997.

LAGO, P. M.; PIVA, J. P.; RAMOS, P. C. R. G.; SFOGGIA, A.; KNIGHT, G.; RAMELET, A.S.; DUNCAN, A. Analgesia e sedação em situações de emergência e unidades de tratamento intensivo pediátrico. J Pediatr (Rio J), 73, 2003.

LECUSSÁN, P. A criança e o diagnóstico. O pediatra deve contar à criança doente seu diagnóstico? Rev. Assoc. Med. Bras. vol.47 no.4 São Paulo Oct./Dec. 2001

MASON, K.P. Challenges in paediatric procedural sedation: political, economic, and clinical aspects, Br J Anaesth, 2014.

MASON, K.P.; LERMAN, J. Review article: dexmedetomidine in children: current knowledge and future applications. Anesth Analg, 113, 2011.

MERKEL, S.I.; VOEPEL-LEWIS, T.; SHAYEVITZ, J.R.; MALVIYA, S. The FLACC: a behavioral scale for scoring postoperative pain in young children. Pediatr Nurs, 23, 1997.

MCCOY, S.; LYTTLE, M.D; HARTSHORN, S.; LARKIN, P.; BRENNER, M.; O'SULLIVAN, R. A qualitative study of the barriers to procedural sedation practices in paediatric emergency medicine in the UK and Ireland. Emerg Med J, 33, 2016.

MIYAKE, R.S.; REIS, A.G.; GRISI, S. Sedation and analgesia for children. Rev Assoc Med Bras, 1998.

RAMALHO, C.E.; BRETAS, P.M., SCHVARTSMAN, C., REIS, A.G. Sedação e analgesia para procedimentos no pronto-socorro de pediatria. J Pediatr (Rio J), 93, 2017.

RANG, H.P.; DALE, M.M.; RITTER, J.M.; FLOWER, Ritter.; HENDERSON, G. Farmacologia, $7^{\mathrm{a}}$ ed., Elsevier, Rio de Janeiro (2012).

ROBACK, M.G.; WATHEN, J.E.; BAJAJ, L., BOTHNER, J.P. Adverse events associated with procedural sedation and analgesia in a pediatric emergency department: a comparison of common parenteral drugs. Acad Emerg Med, 12, 2005.

SAUTER, T.C.; HAUTZ, W.E.; HOSTETTLER, S.; BRODMANN-MAEDER, M.; MARTINOLLI, L.; LEHMANN, B. Interprofessional and interdisciplinary simulation-based training leads to safe sedation procedures in the emergency department. Scand J Trauma Resusc Emerg Med, 24, 2016. 
TACLA, M. T. G. M. Cuidado à criança com dor pós-operatória: experiências de enfermeiras pediatras. Tese (Doutorado), Universidade de São Paulo, Escola de Enfermagem de Ribeirão Preto, 2006.

TOBIAS, J. D., LEDER, M. Procedural sedation: a review of sedative agents, monitoring, and management of complications. Saudi J Anaesth, 5, 2011.

TODD, K. H.; FUNK, K.G.; FUNK, J.P.; BONACCI, R. Clinical significance of reported changes in pain severity. Ann Emerg Med, 27, 1996.

ZAVERI, P.P.; DAVIS, A.B.; O'CONNELL, K.J.; WILLNER, E.; SCHINASI, D.A.; OTTOLINI, M. Virtual reality for pediatric sedation: a randomized controlled trial using simulation. Cureus, 8 , 2016.

WRIGHT, S.W.; CHUDNOFSKY, C.R.; DRONEN, S.C.; KOTHARI, R., BIRRER, P., BLANTON, D.M. Comparison of midazolam and diazepam fr conscious sedation in the emergency department. Ann Emerg Med, 22, 1993.

STANSKI, D.R.; GREENBLATT, D.J.; LOWENSTEIN, E. Kinetics of intravenous and intramuscular morphine. Clin Pharmacol Ther, 24, 1978.

SMITH, D.; CHEEK, H.; DENSON, B.; PRUITT, C.M. Pruitt. Lidocaine pretreatment reduces the discomfort of intranasal midazolam administration: a randomized, double-blind, placebo-controlled trial. Acad Emerg Med, 24, 2017.

SUKYS, G.A.; SCHVARTSMAN, C.; REIS, A.G. Avaliação da sequência rápida de intubação em pronto-socorro pediátrico. J Pediatr (Rio J), 87, 2011.

NOTTERMAN, D.A. Sedation with intravenous midazolam in the pediatric intensive care unit. Clin Pediatr (Phila), 36, 1997.

YOUNG, P.A.; NICOL, M.F.; KENDALL, J.M.; HARRINGTON, A.P. A prospective randomized pilot comparison of intranasal fentanyl and intramuscular morphine for analgesia in children presenting to the emergency department with clinical fractures. Emerg Med, 11, 1999.

\section{Como citar este artigo (Formato ABNT):}

MACHADO, Amanda de Queiroz Germano; VENÂNCIO, Ana Caroliny de Castro; TELES, Francisca Priscila Sampaio Cruz; PASCOAL, Mondeyv de Freitas; MACÊDO, Jacquelinny Lopes de; BATISTA, Hermes Melo Teixeira. Sedação e Analgesia Pediátrica: comentários acerca das drogas utilizadas em procedimentos no pronto-socorro e os cuidados necessários antes, durante e após a sedoanalgesia. Id on Line Rev.Mult. Psic., 2018, vol.12, n.42, Supl. 1, p. 805-822. ISSN: 1981-1179.

Recebido: $14 / 11 / 2018$;

Aceito: $17 / 11 / 2018$ 